\title{
Electronic and spin structure of a family of Sn-based ternary topological insulators
}

\author{
M. G. Vergniory,,$^{1, *}$ T. V. Menshchikova, ${ }^{2}$ I. V. Silkin, ${ }^{2}$ Yu. M. Koroteev, ${ }^{2,3}$ S. V. Eremeev, ${ }^{2,3,4}$ and E. V. Chulkov ${ }^{1,2,4,5}$ \\ ${ }^{1}$ Donostia International Physics Center, P. Manuel de Lardizabal 4, San Sebastián, 20018 Basque Country, Spain \\ ${ }^{2}$ Tomsk State University, pr. Lenina 36, 634050 Tomsk, Russia \\ ${ }^{3}$ Institute of Strength Physics and Materials Science, Siberian Branch, Russian Academy of Sciences, \\ Akademicheskii pr. 2/4, Tomsk, 634021 Russia \\ ${ }^{4}$ St. Petersburg State University, 198504 St. Petersburg, Russia \\ ${ }^{5}$ Departamento de Fisica de Materiales, Facultad de Ciencias Quimicas, UPV/EHU and Centro de Fisica de Materiales, \\ Centro Mixto CSIC-UPV/EHU, Apartado 1072, 20080 San Sebastian/Donostia, Basque Country, Spain
}

(Received 10 June 2015; published 31 July 2015)

\begin{abstract}
We report the bulk and surface electronic properties and spin polarization of a rich family of Sn-based ternary topological insulators studied by means of first-principles calculations within the framework of density functional theory. These compounds exist with the following stoichiometries: $\operatorname{Sn} X_{2} \mathrm{Te}_{4}, \mathrm{Sn}_{4} \mathrm{Te}_{7}$, and $\mathrm{SnBi}_{6} \mathrm{Te}_{10}$ $(X=\mathrm{Sb}$ and $\mathrm{Bi})$. Where a septuple layer or a quintuple layer and septuple layer blocks alternate along the hexagonal axis. We reveal that the bulk band gap in these compounds is about $100 \mathrm{meV}$ and recognize a strong dependence of the spin polarization on the cleavage surface. The calculated spin polarization reaches $85 \%$ in some cases, that is one of the highest predicted values hitherto. Since the electron spin polarization is a relevant parameter for spintronics technology, this new family is suitable for applications within this field.
\end{abstract}

DOI: 10.1103/PhysRevB.92.045134

PACS number(s): 71.20.Nr, 85.75.-d, 03.65.Vf

\section{INTRODUCTION}

Spin-polarized carriers and manipulation of spin currents are essential requirements for spin-based electronics realization. Three-dimensional (3D) $\mathbb{Z}_{2}$ topological insulators (TIs) are characterized by an inverted energy gap at an odd number of symmetry points of the bulk Brillouin zone (BZ) due to spin-orbit coupling (SOC), and as a consequence a spinpolarized metallic surface state (SS) arises in the bulk energy gap displaying unique properties: linear dispersion forming a Dirac cone with a crossing (Dirac) point $\left(E_{D}\right)$ at/close to the Fermi level $\left(E_{F}\right)$ [1-9]. Since time-reversal symmetry is preserved in this type of TI, the surface state is protected from backscattering in the presence of weak perturbations $[1-3,5]$. Owed to these unique properties, these materials are very attractive for spintronics applications $[1-4,10]$. Recently Mellnik et al. [11] demonstrated experimentally that a combination of a standard magnetic material with a TI can be 10 times more efficient for controlling magnetic memory or logic than any other combination of materials discovered to date, making topological spintronics very promising for future technology applications.

Various families of 3D TIs that hold high spin-polarized $\mathrm{SSs}$ have been exhaustively studied, such as the binary $\mathrm{Bi}_{2} \mathrm{Te}_{3}$, $\mathrm{Bi}_{2} \mathrm{Se}_{3}$, and $\mathrm{Sb}_{2} \mathrm{Te}_{3}$ [4,12-21]. These compounds are strong TIs with the topological SS (TSS) mostly located in the first atomic layers adjacent to the vacuum side. However, as was shown by first-principles calculations, the strong spin-orbit entanglement in these materials reduces the spin polarization of the surface states [22]. For instance, $\mathrm{Bi}_{2} \mathrm{Se}_{3}$ possessing a rather wide bulk energy gap supporting the Dirac state was predicted to have only $50 \%$ of spin polarization in the TSS, which turned in good agreement with recent experiment [23].

*maiagv@gmail.com
The in-plane spin polarization of $\mathrm{Bi}_{2} \mathrm{Te}_{3}$ was also measured, and it was predicted to be around $60 \%$ for surfaces [24] and $45 \%$ for thin films [25]. The highest obtained value of these three binary compounds was the one of $\mathrm{Sb}_{2} \mathrm{Te}_{3}$ around $70 \%$ [26], but the Dirac point of the SS, like in the case of $\mathrm{Bi}_{2} \mathrm{Te}_{3}$, lies deep below the Fermi energy and close to the bulk bands, limiting its potential applications. Therefore, the search for new TIs is required in order to achieve the desired properties, crucial for gate control of TI devices [27]: an isolated cone suitable for electronic transport that will display high spin polarization over a wide energy range across $E_{D}$.

An effective way to design new TI materials is with pseudobinary systems like $\mathrm{A}^{\mathrm{IV}} \mathrm{B}^{\mathrm{VI}}-m \mathrm{~A}_{2}^{\mathrm{V}} \mathrm{B}_{3}^{\mathrm{VI}}\left(\mathrm{A}^{\mathrm{IV}}=\mathrm{Ge}\right.$, $\left.\mathrm{Sn}, \mathrm{Pb} ; \mathrm{A}^{\mathrm{V}}=\mathrm{Bi}, \mathrm{Sb} ; \mathrm{B}^{\mathrm{VI}}=\mathrm{Te}, \mathrm{Se}\right)$. These systems are tetradymitelike layered structures but with distinct many layered slabs stacked along the $c$ axis of the hexagonal unit cell; $\mathrm{GeBi}_{2} \mathrm{Te}_{4}, \mathrm{PbBi}_{2} \mathrm{Te}_{4}, \mathrm{PbBi}_{4} \mathrm{Te}_{7}$, and $\mathrm{PbBi}_{6} \mathrm{Te}_{10}$ [28-32] are some of the examples.

Another way of tuning consists of the replacement of the heavy atoms by lighter isoelectronic ones in order to increase the bulk gap. Along these lines a new family of natural sulfur-containing minerals was proposed, with the following compounds predicted to be TIs: $\mathrm{PbBi}_{2} \mathrm{Te}_{2} \mathrm{~S}_{2}, \mathrm{~Pb}_{2} \mathrm{Bi}_{2} \mathrm{Te}_{2} \mathrm{~S}_{3}$, $\mathrm{PbBi}_{4} \mathrm{Te}_{4} \mathrm{~S}_{3}$ [33]. The later family displays a spin polarization between $70 \%$ and $80 \%$, one of the highest predicted up to now together with the one of $\mathrm{Bi}_{2} \mathrm{Se}_{2} \mathrm{Te}$ and $\mathrm{Bi}_{2} \mathrm{Te}_{2} \mathrm{Se}$ [34] with a maximum of $77 \%$.

In this work we present detailed $a b$ initio calculation of the electronic and spin structure of two compounds that have not been reported yet, $\mathrm{SnSb}_{4} \mathrm{Te}_{7}$ and $\mathrm{SnBi}_{6} \mathrm{Te}_{10}$, which are strong $3 \mathrm{D} \mathbb{Z}_{2}$ TIs. They belong to a wider family of already predicted TIs, which displays different stoichiometries, such as $\mathrm{Sn} X_{2}^{\mathrm{IV}} \mathrm{Te}_{4}$ or $\mathrm{Sn} X_{4}^{\mathrm{IV}} \mathrm{Te}_{7}(X=\mathrm{Sb}$ and $\mathrm{Bi})$ [35]. Even though the surface electronic structure of some of these compounds was published earlier, the analysis of the spin polarization is still lacking. Due to the relevance of the spin polarization in 
TABLE I. Lattice parameters and gap width (indirect) for the Sn-family ternary compounds.

\begin{tabular}{lccc}
\hline \hline Compound & $a(\AA)$ & $c(\AA)$ & Gap (eV) \\
\hline $\mathrm{SnBi}_{2} \mathrm{Te}_{4}$ & 4.39 & 41.61 & 0.015 \\
$\mathrm{SnSb}_{2} \mathrm{Te}_{4}$ & 4.29 & 41.55 & 0.16 \\
$\mathrm{SnSb}_{4} \mathrm{Te}_{7}$ & 4.37 & 23.77 & 0.11 \\
$\mathrm{SnBi}_{4} \mathrm{Te}_{7}$ & 4.39 & 23.99 & 0.07 \\
$\mathrm{SnBi}_{6} \mathrm{Te}_{10}$ & 4.39 & 102.59 & 0.095 \\
\hline \hline
\end{tabular}

the field, this analysis will be provided in this paper as well. In particular, $\mathrm{SnSb}_{4} \mathrm{Te}_{7}$ and $\mathrm{SnBi}_{6} \mathrm{Te}_{10}$ present ordered stable phases and a band gap around $100 \mathrm{meV}$. We will also show that the electronic structure of these compounds can be tuned by considering different cleaved surfaces, and in particular some of the surface terminations can reach a spin polarization of almost $80 \%$.

The present article is organized as follows. Section II provides computational details. Sections II and III present a substantial study of the electronic band structure and spin polarization of $\mathrm{SnSb}_{4} \mathrm{Te}_{7}$ and $\mathrm{SnBi}_{6} \mathrm{Te}_{10}$. We will end with the summary and conclusions in Sec. IV.

\section{CALCULATION METHODS}

The structural optimization and electronic calculations were performed within the density functional formalism implemented in VASP [36,37]. The interaction between ion cores and valence electrons was treated by the projector augmented wave method $[38,39]$. To describe the exchange-correlation energy, we used the generalized gradient approximation [40]. The Hamiltonian contained the scalar relativistic corrections and the spin-orbit coupling was taken into account by the second variation method [41]. The DFT-D2 method of Grimme [42] was used for taking van der Waals ( $\mathrm{vdW}$ ) forces into account.

We used experimental lattice parameters with optimized atomic positions in the unit cell (see Table I). The $7 \times 7 \times 7$ and $11 \times 11 \times 1 k$-point grids for bulk and slab self-consistent calculations, respectively, were used. The kinetic energy cutoff for the plane-wave basis set was $220 \mathrm{eV}$. To simulate the (0001) surfaces we used over 40 atomic layers to avoid the interactions between surfaces. The number of atomic layers will be specified for each compound.

\section{RESULTS AND DISCUSSION}

\section{A. $\mathrm{SnSb}_{2} \mathrm{Te}_{4}$ and $\mathrm{SnBi}_{2} \mathrm{Te}_{4}$}

The hexagonal lattice of $\operatorname{Sn}_{2} \mathrm{Te}_{4}(X=\mathrm{Sb}, \mathrm{Bi})$ can be represented as a sequence of septuple layer (SL) blocks that alternate in a series along the hexagonal $c$ axis [see Fig. 1(a)]. Since the bulk and surface electronic structures of these TIs have been already studied (see Refs. [43,44]), we adduce in detail here only quantitative analysis of the spin polarization of the topological SS which was not reported for these compounds.

In Figs. 2(a) and 2(b) one can observe spin-resolved surface band spectra for $\mathrm{SnSb}_{2} \mathrm{Te}_{4}$ and $\mathrm{SnBi}_{2} \mathrm{Te}_{4}$ with a spin-helical

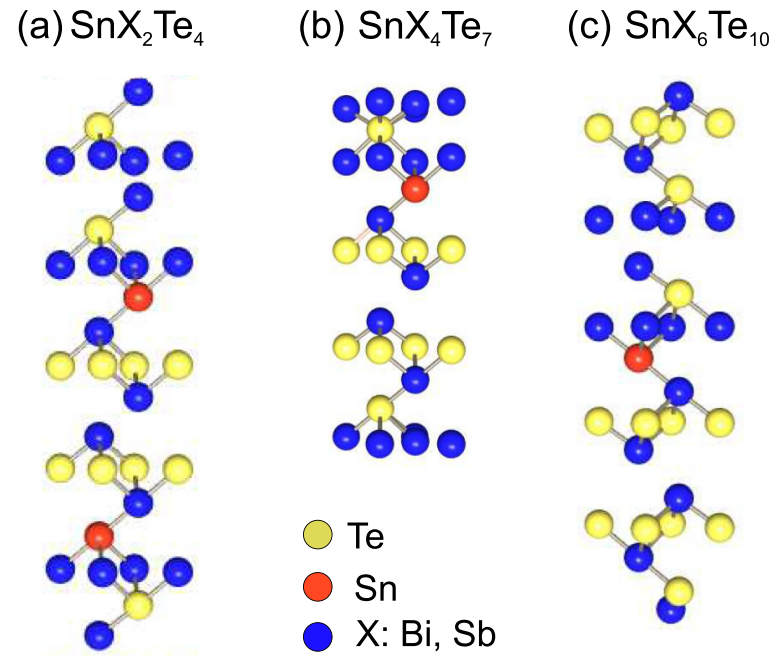

FIG. 1. (Color online) Atomic structure of Sn-based ternary compounds (a) $\mathrm{Sn}_{2} \mathrm{Te}_{4}$, (b) $\operatorname{Sn} X_{4} \mathrm{Te}_{7}$, and (c) $\operatorname{Sn} X_{6} \mathrm{Te}_{10}$ $(X=\mathrm{Sb}, \mathrm{Bi})$

Dirac surface state residing in the band gap. Figures 2(c) and 2(d) show in-plane spin polarization of the TSS of the TSS (where $100 \%$ correspond to $|S|=\frac{1}{2}$ ) for $\bar{\Gamma}-\bar{M}$ direction. In both compounds the spin polarization is maximal near the Dirac point and it decreases with $k_{\|}$when approaching the bottom of the conduction band. The same dependence of the in-plane spin polarization on $k_{\|}$is observed along $\bar{\Gamma}-\overline{\mathrm{K}}$ direction. In the case of $\mathrm{SnSb}_{2} \mathrm{Te}_{4}$ spin polarization in the vicinity of the $\bar{\Gamma}$ point is $\sim 65 \%$ and a weak dependence of the spin polarization on the wave vector takes place. For $\mathrm{SnBi}_{2} \mathrm{Te}_{4}$ the maximal spin polarization reaches $\sim 75 \%$ and decreases by factor of 1.5 at $k_{\|} \approx 0.08 \AA^{-1}$ after which it varies slightly up to the conduction band minimum. Along with this, $S_{z}$ spin component increases with distance from the Dirac point which is well correlated with progressive hexagonal warping in the Dirac cone (see Ref. [45]).

Same trends of the spin polarization as a function of the wave vector are observed in the binary quintuplelayer-structured (QL-structured) TIs, $\mathrm{Sb}_{2} \mathrm{Te}_{3}$ and $\mathrm{Bi}_{2} \mathrm{Te}_{3}$ [see Figs. 2(c) and 2(d)], however, the values of $S_{x}$ are as a whole smaller than in SL-structured $\mathrm{SnSb}_{2} \mathrm{Te}_{4}$ and $\mathrm{SnBi}_{2} \mathrm{Te}_{4}$, especially at small $k_{\|}$. As one can see in Figs. 2(a) and 2(b) (right subpanels) near the Dirac point the TSS is spread mainly in the surface SL; however, it has a noticeable weight in the second SL. Moving away from the Dirac point (DP) the weight of the TSS in deeper atomic layers decreases and the state becomes predominantly localized in the near-surface layers. It should be noted that similar dependence of the TSS localization was found earlier in $\mathrm{Bi}_{2} \mathrm{Te}_{3}$ Ref. [46]. This change in the TSS localization leads to the fact that at large wave vectors the contribution of deeper layers to the total spin polarization decreases and it causes the observed decline in the polarization a as function of $k_{\|}$. In the case of $\mathrm{SnBi}_{2} \mathrm{Te}_{4}$ the charge-density profile at $k_{\|}>k_{2}$ remains almost invariable, as a consequence we observe the nearly flat behavior of the polarization at large wave vectors. 
(a)

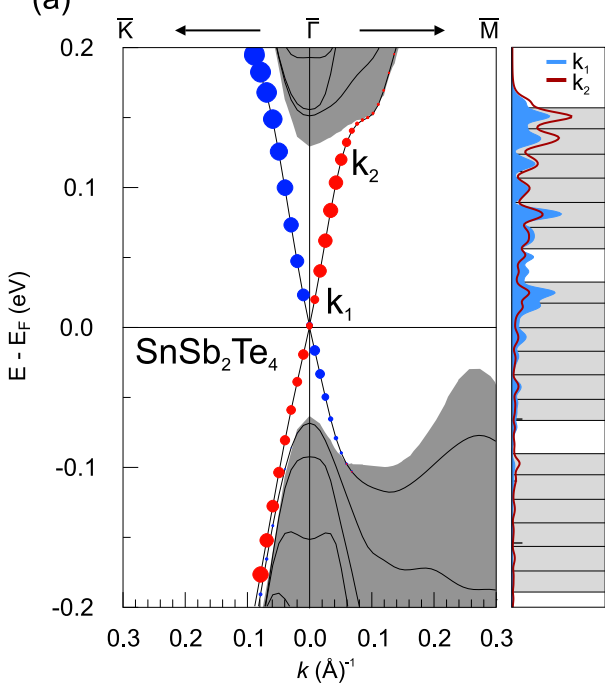

(b)

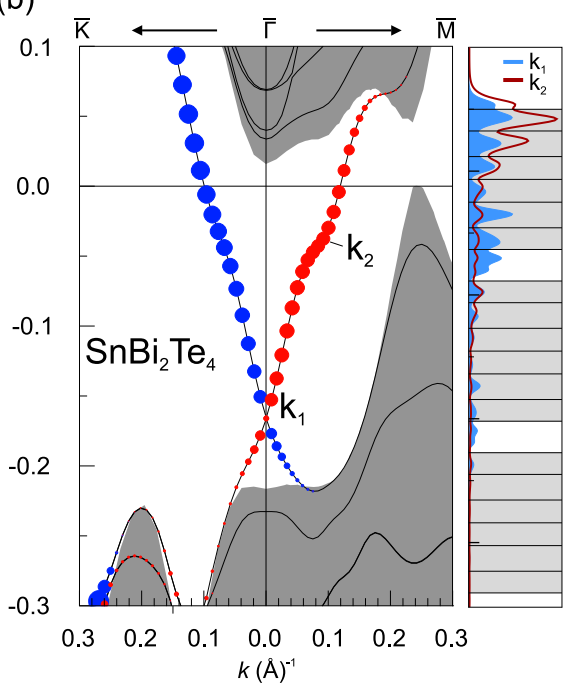

(c)

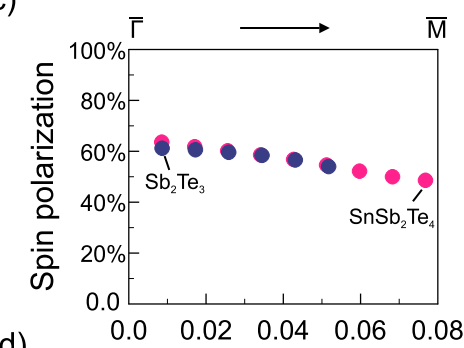

(d)

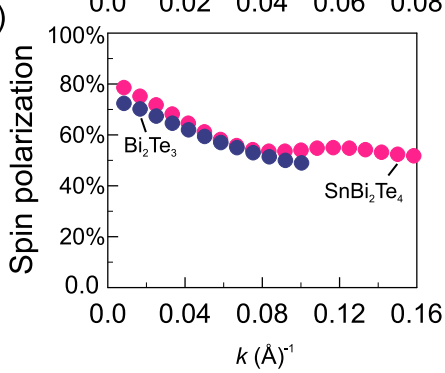

FIG. 2. (Color online) (a) and (b) Left subpanels: Band spectrum of the $\mathrm{SnSb}_{2} \mathrm{Te}_{4}$ and $\mathrm{SnBi}_{2} \mathrm{Te}_{4}$ (0001) surfaces (black lines) and the projected bulk band structure (gray), respectively. Size of the color circles represent weights of the state in the surface SL block multiplied by values of in-plane spin components $S_{x}$ and $S_{y}$ (red and blue colors denote positive and negative projection of spin vector $\vec{S}$ on Cartesian axes, respectively). Right subpanels in (a) and (b) show charge density of the TSS integrated over $x, y$ at the respective $k$ points in the left subpanels. Vertical lines indicate atomic positions. (c) and (d) Spin-polarization values as a function of the wave vector for the upper part (above the Dirac point) of the TSS in $\mathrm{SnSb}_{2} \mathrm{Te}_{4}$ and $\mathrm{SnBi}_{2} \mathrm{Te}_{4}$, respectively.

\section{B. $\mathrm{SnSb}_{4} \mathrm{Te}_{7}$ and $\mathrm{SnBi}_{4} \mathrm{Te}_{7}$}

The crystal structure of $\mathrm{SnSb}_{4} \mathrm{Te}_{7}$ (as well as of $\mathrm{SnBi}_{4} \mathrm{Te}_{7}$ ) is hexagonal (group $P \overline{3} m 1$ ) and the unit cell contains alternating quintuple and septuple layer blocks formed by alternating anion and cation layers, Te-Sb-Te-Sb-Te and Te-Sb-Te-Sn-Te$\mathrm{Sb}-\mathrm{Te}$, respectively [35] [see Fig. 1(b)]. Each block consists of strongly bonded ionic-covalent atoms, whereas the bonding between blocks is determined by vdW forces.

As can be seen in Fig. 3(a), $\mathrm{SnSb}_{4} \mathrm{Te}_{7}$ is a semiconductor with an indirect bulk band gap of $\sim 100 \mathrm{meV}$. An analysis of the orbital composition of the states at the edges of the band gap shows that there is a complicated SOC-induced inversion of $\mathrm{Sb}$ and Te states near $A$ point. Indeed, the local density of states (LDOS) without (w/o) SOC-included demonstrates that the top of the valence band is predominantly formed by Te atom states, while the bottom of the conduction band is composed of $\mathrm{Sb}$ and Sn orbitals of both SL and QL blocks, respectively [Fig. 3(b), left panel]. The SOC inclusion causes band gap broadening and induces inversion of states. However, the top valence band is determined by the states localized in $\mathrm{Sn}$ and $\mathrm{Sb}$ atoms of SL blocks, whereas Sb states of QL blocks lie below the bulk gap edge [Fig. 3(b), right panel]. The lower conduction band is mostly composed of Te orbitals of QL block. Such intriguing SOC-induced band inversion appears due to the complexity of the crystal structure. Note, almost similar band inversion was also found for a Pb-based isostructural system [28,29], where small differences took place: the bottom of the conduction band is mostly formed by Te states of both SL and QL blocks.

The bulk and surface electronic structures of $\mathrm{SnBi}_{4} \mathrm{Te}_{7}$ have been already reported [30], thus we will focus on the spin polarization of the topological SS for this compound.

Owing to the topological nature of these compounds their surface should hold gapless TSS. Because the crystal structure of $\mathrm{SnSb}_{4} \mathrm{Te}_{7}$ compound is constituted by alternating SL and QL building blocks, the surfaces formed after cleavage of this crystal have two possible terminations. The surface can be terminated by SL or QL block which we will denote as 7L and $5 \mathrm{~L}$ surfaces, respectively. The $7 \mathrm{~L}$ and $5 \mathrm{~L}$ surfaces were simulated by 43 atomic (SL-QL-SL-QL-SL-QL-SL) and 41 atomic (QL-SL-QL-SL-QL-SL-QL) layer slabs, respectively.

Figures 4(a) and 4(b) and 4(e) and 4(f) show the band structure of the 7L and 5L surfaces of $\mathrm{SnSb}_{4} \mathrm{Te}_{7}$ and $\mathrm{SnBi}_{4} \mathrm{Te}_{7}$, respectively. In $\mathrm{SnSb}_{4} \mathrm{Te}_{7}$ at the 7L surface the TSS dispersion is strongly linear above and below the DP, while in the $5 \mathrm{~L}$ case the TSS deviates from linear dispersion in the vicinity of the DP. The Dirac cone at the $7 \mathrm{~L}$ surface of $\mathrm{SnBi}_{4} \mathrm{Te}_{7}$ is warped near the conduction band and the TSS dispersion at the $5 \mathrm{~L}$ surface is in general similar to that at the $5 \mathrm{~L}$ surface of $\mathrm{SnSb}_{4} \mathrm{Te}_{7}$. Another interesting feature of the $5 \mathrm{~L}$ surface spectrum of $\mathrm{SnSb}_{4} \mathrm{Te}_{7}\left(\mathrm{SnBi}_{4} \mathrm{Te}_{7}\right)$ is the occupied Dirac cone which lies at $-0.15 \mathrm{eV}(-0.25 \mathrm{eV})$ below the Fermi energy. This is the surface state that survives from $\mathrm{Sb}(\mathrm{Bi})_{2} \mathrm{Te}_{3}$, having the dispersion and localization identical to the Dirac state of the parent compound.

The TSS charge density distribution of the $5 \mathrm{~L}$ and $7 \mathrm{~L}$ surfaces differs. While the $7 \mathrm{~L}$ surface of $\mathrm{SnSb}_{4} \mathrm{Te}_{7}$ is mostly localized in two blocks (surface QL and subsurface SL), in the case of the $5 \mathrm{~L}$ surface in the vicinity of $\bar{\Gamma}$ it is predominantly localized within the subsurface SL block and partly in the surface QL block and third QL. On the contrary, at the 7L surface of $\mathrm{SnBi}_{4} \mathrm{Te}_{7}$ the TSS lies completely in the surface SL like in the case of $\mathrm{PbBi}_{4} \mathrm{Te}_{7}[28,29]$, whereas the 5L surface TSS in $\mathrm{SnBi}_{4} \mathrm{Te}_{7}$ displays a localization similar to that at the $5 \mathrm{~L}$ surface of $\mathrm{SnSb}_{4} \mathrm{Te}_{7}$.

Figures 4(c) and 4(d) and 4(g) and 4(h) show the in-plane spin polarization of the TSS at $7 \mathrm{~L}$ and $5 \mathrm{~L}$ surfaces of 

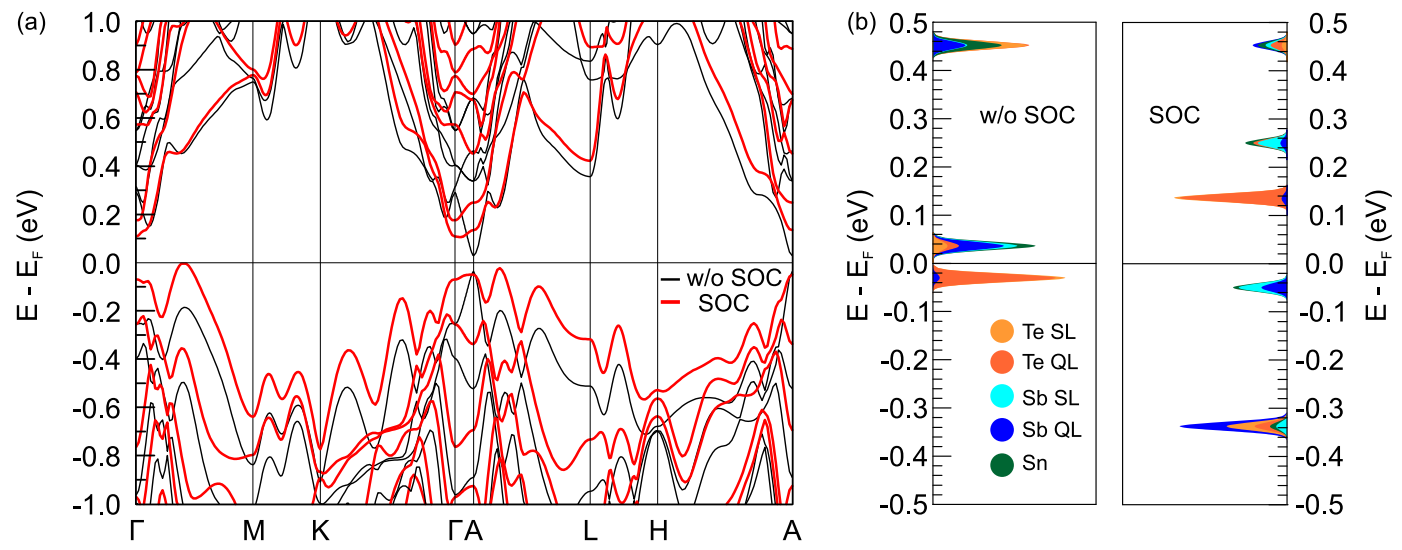

FIG. 3. (Color online) (a) Bulk band spectra of $\mathrm{SnSb}_{4} \mathrm{Te}_{7}$ along the high symmetry lines in the Brillouin zone. Red/black lines correspond to results with/without SOC included. (b) LDOS calculated without (left side) and with (right side) SOC included at the vicinity of the $A$ point. Colors in the local density of states correspond to different atomic contributions.

(a)

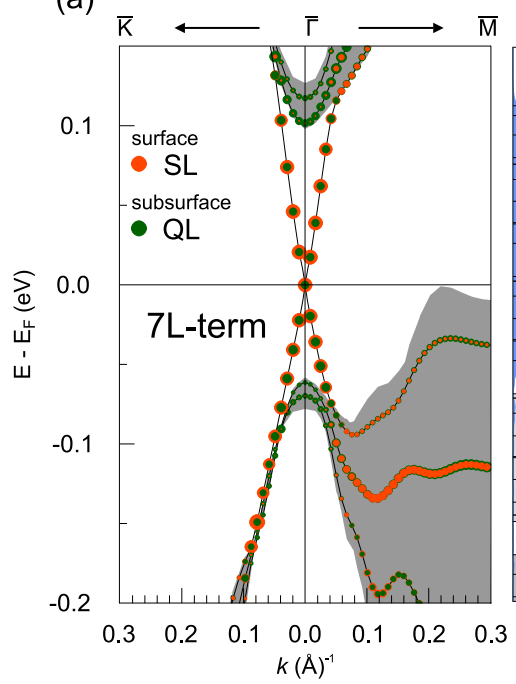

(e)
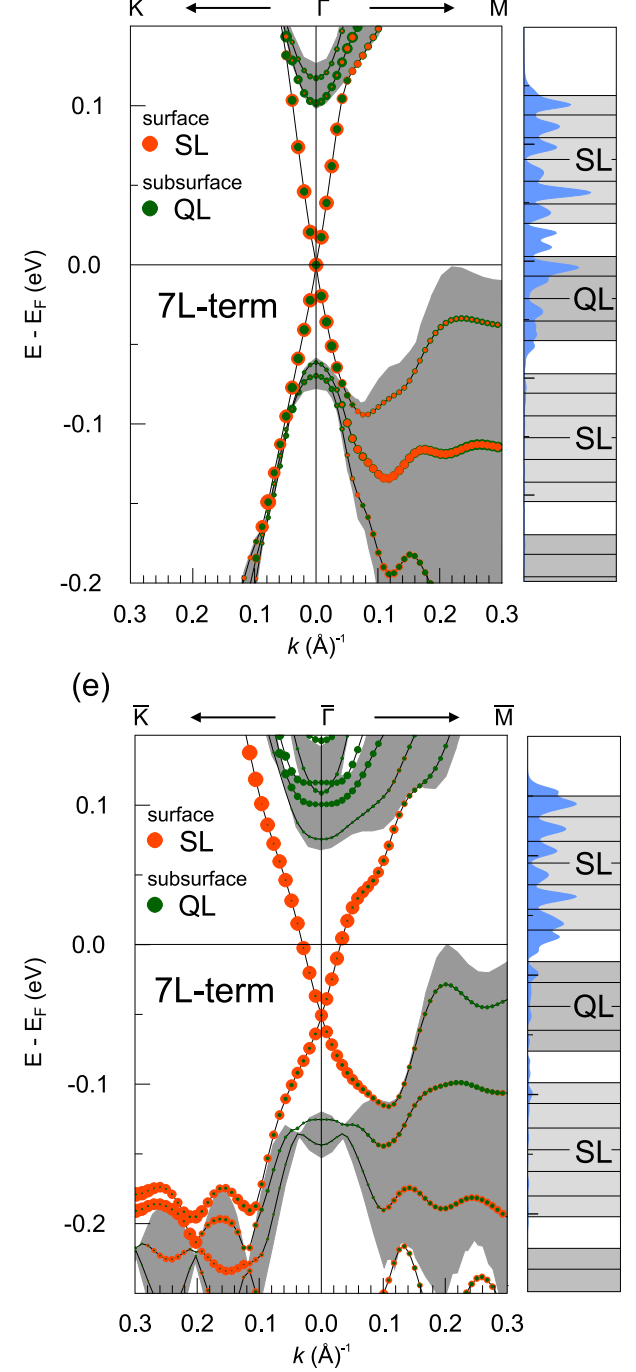

(b)

(f)

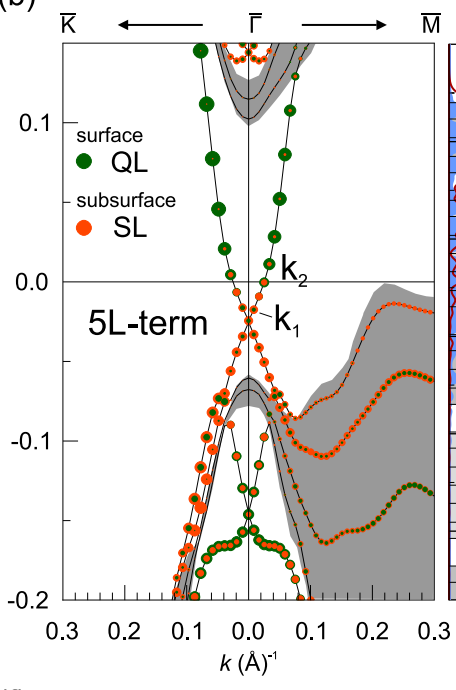

(f)

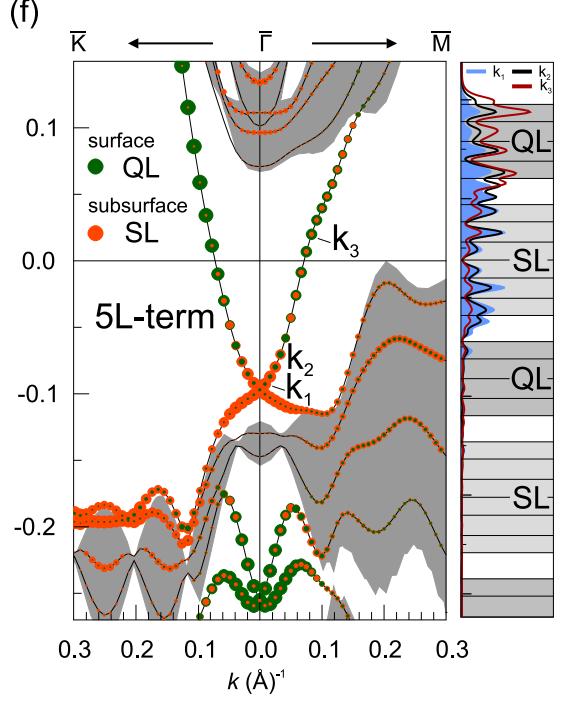

(c)
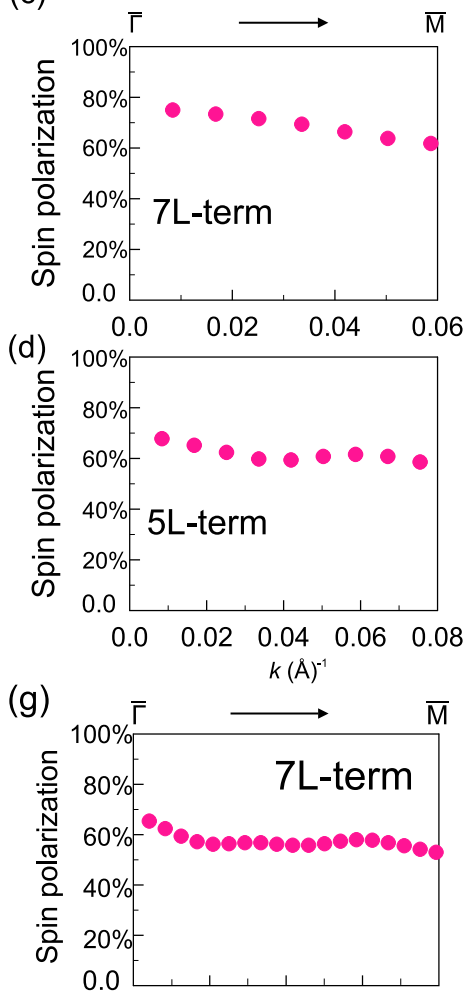

(h)

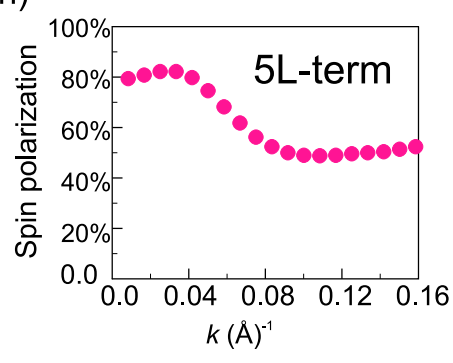

FIG. 4. (Color online) Band spectra and charge density profiles for $7 \mathrm{~L}$ (a) and $5 \mathrm{~L}$ (b) surfaces of $\mathrm{SnSb}_{4} \mathrm{Te}_{7}$ and for $7 \mathrm{~L}$ (e) and $5 \mathrm{~L}$ (f) surfaces of $\mathrm{SnBi}_{4} \mathrm{Te}_{7}$. Spin polarization as a function of the wave vector for the upper part of the TSS at 7L (c) and (g) and 5L (d) and (h) surfaces of $\mathrm{SnSb}_{4} \mathrm{Te}_{7}$ and $\mathrm{SnBi}_{4} \mathrm{Te}_{7}$, respectively. 

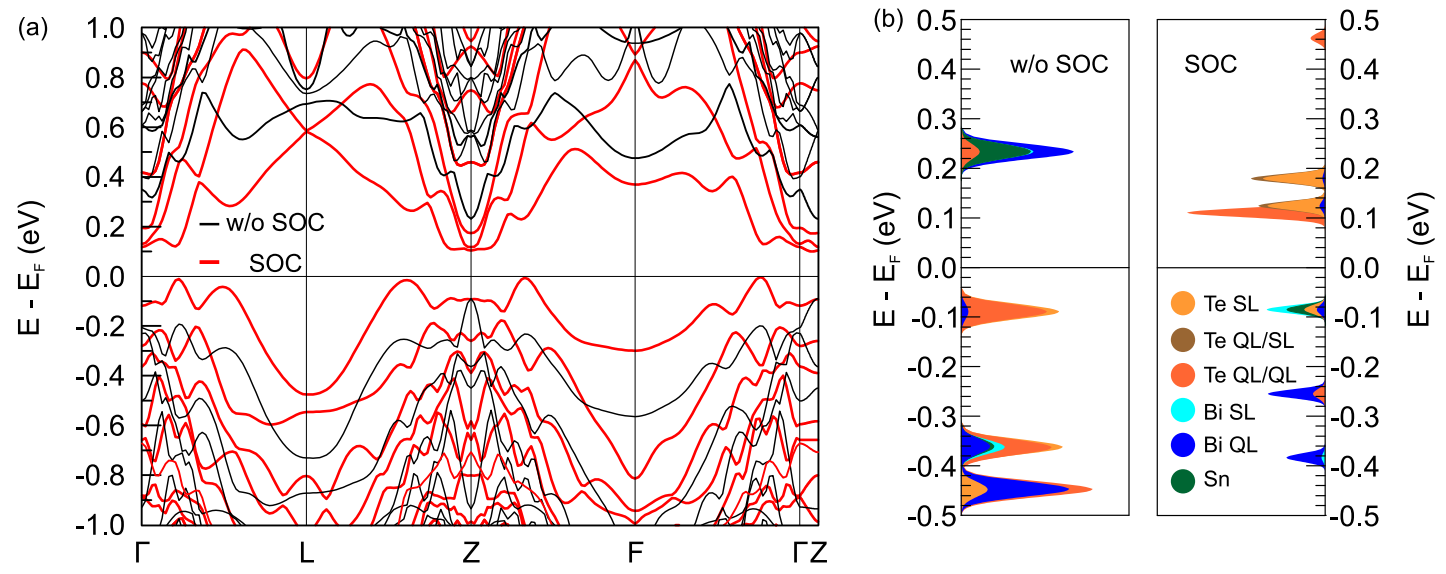

FIG. 5. (Color online) Same as in Fig. 3 but for $\mathrm{SnBi}_{6} \mathrm{Te}_{10}$.

$\mathrm{SnSb}_{4} \mathrm{Te}_{7}$ and $\mathrm{SnBi}_{4} \mathrm{Te}_{7}$. As one can observe for 7L surfaces the dependence of the polarization on $k_{\|}$has a maximum in the vicinity of the DP and as a whole is similar to that for $\mathrm{SnSb}_{2} \mathrm{Te}_{4}$ and $\mathrm{SnBi}_{2} \mathrm{Te}_{4}$. However, at $5 \mathrm{~L}$ surfaces the situation is more intriguing. The spin polarization at $k_{\|}=0.04 \AA^{-1}$ has a minimum for $\mathrm{SnSb}_{4} \mathrm{Te}_{7}$ TSS while the $\mathrm{SnBi}_{4} \mathrm{Te}_{7}$ TSS has a maximum approximately at the same $k_{\|}$reaching $82 \%$. This interesting behavior is related to the change in the spatial localization of the Dirac state. In Fig. 4(b) one can observe that at $k_{\|}=k_{2}$ the localization of the TSS in $\mathrm{SnSb}_{4} \mathrm{Te}_{7}$ changes from mainly subsurface SL to the surface $\mathrm{QL}$ and therefore makes the localization region thinner, while in $\mathrm{SnBi}_{4} \mathrm{Te}_{7}$ the TSS being mostly localized in the subsurface SL at small $k_{\|}$becomes widely localized within surface QL and subsurface SL near $k_{\|}=0.04 \AA^{-1}\left(k_{2}\right)$ causing the increase in the in-plane spin polarization. This feature has an advantage: the maximal spin polarization in the TSS arises far from the DP, at the point where the density of states involved in spin-polarized transport is increased.

\section{C. $\mathrm{SnBi}_{6} \mathrm{Te}_{10}$}

Ternary compounds $\operatorname{Sn} X_{6} \mathrm{Te}_{10}(X=\mathrm{Sb}, \mathrm{Bi})$ have also a tetradymitelike crystal structure but it differs from $\mathrm{Sn} X_{4} \mathrm{Te}_{7}$ in the following way, it is built alternating SL and two QL blocks along the hexagonal $c$ axis [Fig. 1(c)]. To determine the $\mathbb{Z}_{2}$ topological invariant we checked the parity of the wave functions at TRIM of the bulk valence band spectrum [Fig. 5(a)] and found that owing to the inversion at $Z$ point the invariant is nontrivial for $\mathrm{SnBi}_{6} \mathrm{Te}_{10}$, while $\mathrm{SnSb}_{6} \mathrm{Te}_{10}$ was found to be a trivial insulator. As can be seen in Fig. 5(b) the SOC-induced band inversion in $\mathrm{SnBi}_{6} \mathrm{Te}_{10}$ is in general similar to that observed in $\mathrm{SnSb}_{4} \mathrm{Te}_{7}$, however, there are some peculiarities due to its more complex crystal structure. The lowest conduction band at $Z$ is formed by orbitals of Te atoms lying on the border between QLs [marked as Te QL/QL in Fig. 5(b), right panel] while the next two conduction bands are composed of hybridized states localized on Te atoms of QL located on the border between QL and SL blocks (marked as Te QL/SL) and on edge Te atoms of SL block (Te SL).

The $\mathrm{SnBi}_{6} \mathrm{Te}_{10}$ crystal has two nonequivalent cleavage planes: between SL and QL blocks and between two adjacent
QLs, resulting in three different surface terminations. Two of them, $5 \mathrm{~L}$ and $7 \mathrm{~L}$, are similar to those in $\mathrm{SnBi}_{4} \mathrm{Te}_{7}$. The former has QL block on top and SL block beneath it and in the latter one the sequence of surface and subsurface blocks is inverted. The third termination, which is absent in the $\mathrm{SnBi}_{4} \mathrm{Te}_{7}$ compound with a simpler crystal structure, is the surface terminated by two successive QL blocks, which we will call 5-5L termination. In the calculations 7L, 5L, and 5-5L terminations were simulated by 41,44 , and 51 atomic layer slabs, respectively. Figures 5(a)-5(c) show the band structure of surfaces with all types of terminations.

The dispersion of Dirac cones at $7 \mathrm{~L}$ and $5 \mathrm{~L}$ surfaces is similar to the respective surfaces of $\mathrm{SnBi}_{4} \mathrm{Te}_{7}$ as well as to all terminations found for $\mathrm{PbBi}_{6} \mathrm{Te}_{10}$ [29]. In particular, like in $\mathrm{PbBi}_{6} \mathrm{Te}_{10}$ we find that owing to specific bulk band inversion the DP at the 5-5L surface lies in the local gap of the bulk valence band [Fig. 6(c)].

Likewise, the charge distribution of the topological state at $7 \mathrm{~L}$ and $5 \mathrm{~L}$ surfaces of $\mathrm{SnBi}_{6} \mathrm{Te}_{10}$ demonstrate a similar behavior as at the surfaces of $\mathrm{SnBi}_{4} \mathrm{Te}_{7}$. Thus, at the $7 \mathrm{~L}$ surface the TSS is localized in the surface SL block and its maximum gradually shifts towards the near-surface atomic layers as we move away from the DP, which is responsible for the moderate dependence of the spin polarization on the wave vector [Fig. 6(d)], with a maximum near the center of the Brillouin zone $(\sim 65 \%)$ and a minimum near the bottom of the conduction band $(53 \%)$. At the 5L surface the TSS being strongly localized in the subsurface block, in the vicinity of DP, relocates to the surface QL block with $k_{\|}$increasing in the same way as at the 5L surface of $\mathrm{SnBi}_{4} \mathrm{Te}_{7}$ and, as one can see in Fig. 6(e), the maximum of the spin polarization $(\sim 84 \%)$ is located at $k_{\|} \approx$ $0.03 \AA^{-1}$, similar to the case of $5 \mathrm{~L}-\mathrm{SnBi}_{4} \mathrm{Te}_{7}$ [see Fig. 4(h)].

The dispersion and shape of the Dirac cone at the 5-5L surface are very similar to the ones found for $\mathrm{PbBi}_{6} \mathrm{Te}_{10}$ [29]. Particularly, the Dirac point is located in the local gap of the bulk valence band. As a result, the Dirac cone possesses two segments: in the local and in the fundamental band gap. However, the charge density of the SS in both segments is mostly localized in the surface QL block. As can be seen in Fig. 6(f) the total in-plane spin component $S_{x}$ at small $k_{\|}$is maximal near $\bar{\Gamma}$ point and decreases in the direction of the bulk band continuum [marked by the gray rectangle in Fig. 6(f)] reproducing the spin polarization behavior of 
(a)

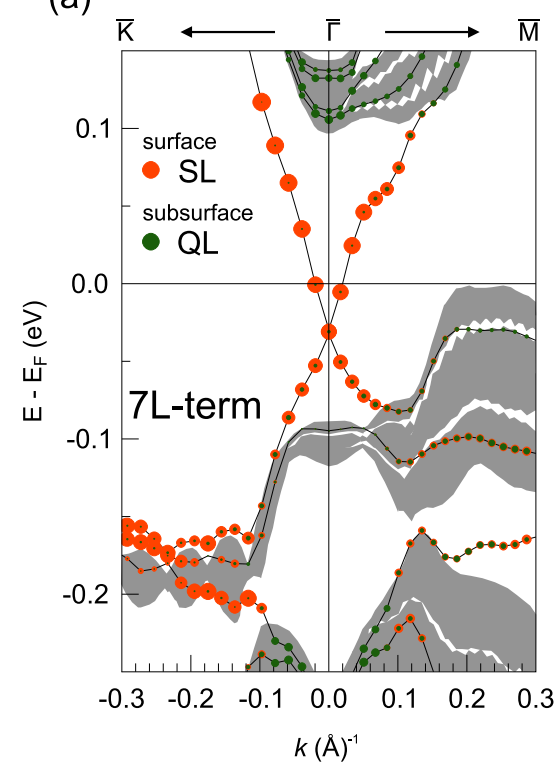

(d)

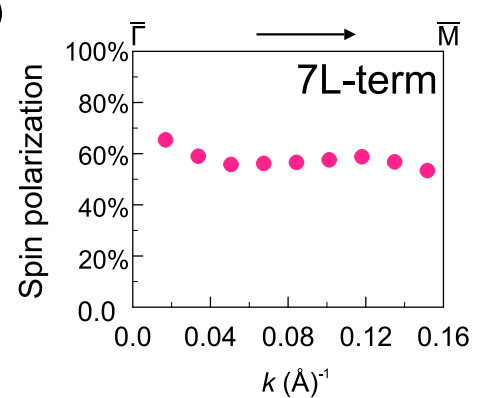

(b)

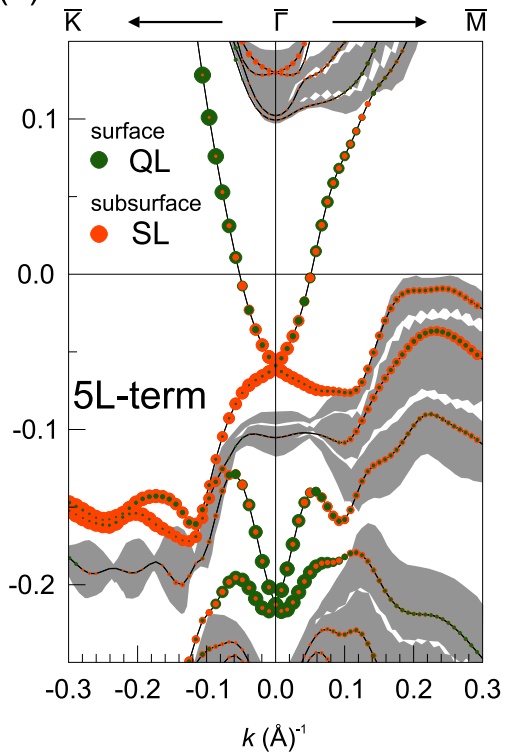

(e)

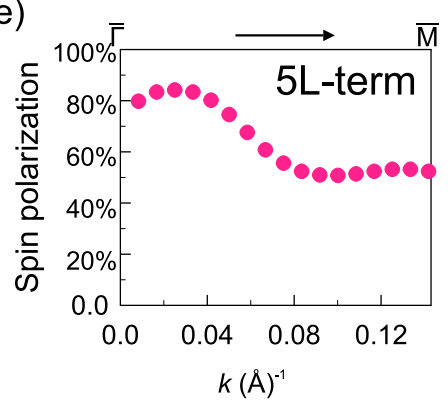

(c)

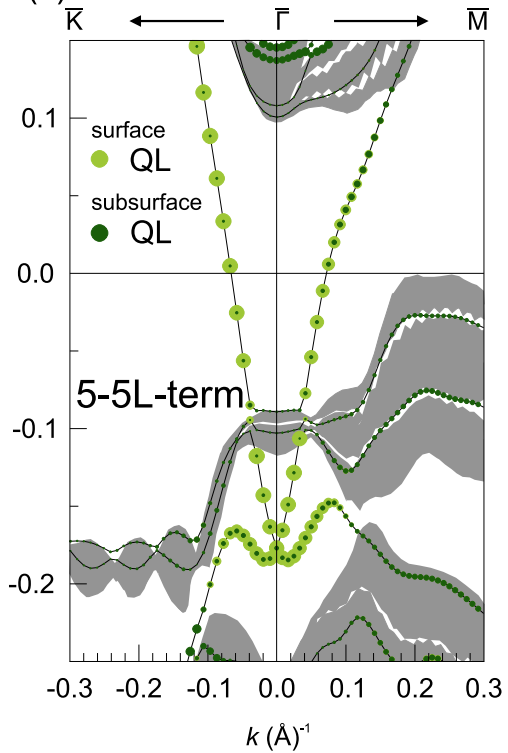

(f)

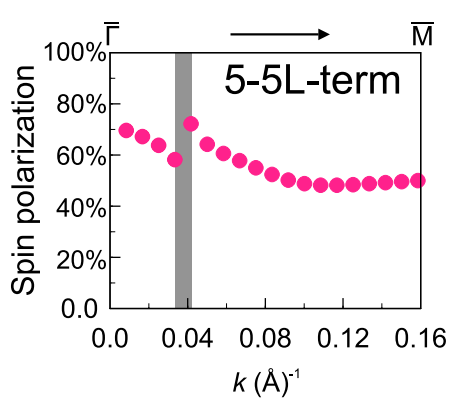

FIG. 6. (Color online) (a)-(c) Band spectrum of the $\mathrm{SnBi}_{6} \mathrm{Te}_{10}$ surface terminated by SL, QL, and double QL blocks, respectively. The size of the color circles reflects the weight of the state in surface and subsurface blocks. (d)-(f) Spin polarization in the momentum space for the upper part of the TSS (above the DP). The gray narrow rectangle in (f) marks the $k_{\|}$region where TSS crosses the bulk valence band continuum.

$\mathrm{Bi}_{2} \mathrm{Te}_{3}$ [Fig. 2(d)]. The same dependence of the in-plane spin component on $k_{\|}$takes place in the fundamental band gap. The maximal spin polarization in both segments reaches $\sim 70 \%$.

\section{SUMMARY AND CONCLUSION}

It has been established that $\mathrm{Sn}$-based ternary compounds $\mathrm{Sn} X_{2} \mathrm{Te}_{4}, \mathrm{Sn} X_{4} \mathrm{Te}_{7}$, and $\mathrm{SnBi}_{6} \mathrm{Te}_{10}(X=\mathrm{Sb}, \mathrm{Bi})$ are 3D $\mathbb{Z}_{2}$ topological insulators while $\mathrm{SnSb}_{6} \mathrm{Te}_{10}$ is not. Since $\mathrm{Sn} X_{4} \mathrm{Te}_{7}$ and $\mathrm{SnBi}_{6} \mathrm{Te}_{10}$ have a more complicated crystal structure compared to binary TIs they demonstrate strong differences in the dispersion of the topological surface state and its spin polarization depending on the cleavage surface. In particular, 7L surfaces in $\mathrm{Sn} X_{4} \mathrm{Te}_{7}$ and $\mathrm{SnBi}_{6} \mathrm{Te}_{10}$ are characterized by a maximal spin polarization in the vicinity of the Dirac point and its weak dependence on the wave vector. The latter fact allows us to increase the carrier density (by means of the upward shift of the Fermi level) involved in the transport experiments for a wide energy range without a significant decrease in the spin polarization. For the $5 \mathrm{~L}$ surface terminations the situation is more intriguing. With the exception of $\mathrm{SnSb}_{4} \mathrm{Te}_{7}$, the maximum of the in-plane spin polarization is positioned far from the Dirac point owing to the change in the spatial localization of the Dirac state. This feature allows us to realize the regime of the spin-polarized transport with increased density of state which is accompanied by maximal spin polarization. The $7 \mathrm{~L}$ surface of the $\mathrm{SnSb}_{4} \mathrm{Te}_{7}$ and $5 \mathrm{~L}$ surfaces of the $\mathrm{SnBi}_{4} \mathrm{Te}_{7}$ and $\mathrm{SnBi}_{6} \mathrm{Te}_{10}$ seem to be more suitable systems for spintronics applications due to high spin polarization. These findings will lead us to realize a proper choice for spintronics applications.

\section{ACKNOWLEDGMENTS}

We acknowledge stimulating discussions with M. M. Otrokov during the elaboration of the manuscript. This work is supported by a grant (No. 8.1.05.2015) from The Tomsk State University Academic D. I. Mendeleev Fund Program and Russian Foundation for Basic Research (Grant 13-02-12110 ofi_m). M.G.V. and E.V.C. acknowledge partial support from the Basque Country Government, Departamento de Educación, Universidades e Investigación (Grant No. IT756-13), the Spanish Ministerio de Economía e Innovación (Grants No. FIS2010-19609-C02-01 and No. FIS2013-48286C2-1-P), and the FEDER funding and Saint Petersburg State University (Project No. 11.50.202.2015 ). Calculations were performed at the DIPC computing center of the Basque Country (Spain) and at the SKIF-Cyberia supercomputer of Tomsk State University (Russian Federation). 
[1] L. Fu and C. L. Kane, Phys. Rev. B 76, 045302 (2007).

[2] J. C. Y. Teo, L. Fu, and C. L. Kane, Phys. Rev. B 78, 045426 (2008).

[3] X.-L. Qi, T. L. Hughes, and S. C. Zhang, Phys. Rev. B 78, 195424 (2008).

[4] H. Zhang, C. X. Liu, X. L. Qi, Z. Fang, and S. C. Zhang, Nat. Phys. 5, 438 (2009).

[5] M. Z. Hasan and C. L. Kane, Rev. Mod. Phys. 82, 3045 (2012).

[6] D. Pesin and A. H. Mcdonald, Nat. Mater. 11, 409 (2010).

[7] J. E. Moore, Nature (London) 464, 194 (2010).

[8] S. V. Eremeev, G. Bihlmayer, M. Vergniory, Y. M. Koroteev, T. V. Menshikova, J. Henk, A. Ernst, and E. V. Chulkov, Phys. Rev. B 83, 205129 (2011).

[9] S. V. Eremeev, T. V. Menshchikova, I. V. Silkin, M. G. Vergniory, P. M. Echenique, and E. V. Chulkov, Phys. Rev. B 91, 245145 (2015).

[10] Y. Xia, D. Qian, D. Hsieh, L. Wray, A. Pal, H. Lin, A. Bansil, D. Grauer, Y. S. Hor, R. J. Cava, and M. Z. Hasan, Nat. Phys. 5, 398 (2009).

[11] A. R. Mellnik, J. S. Lee, A. Richardella, J. L. Grab, P. J. Mintun, M. H. Fischer, A. Vaezi, A. Manchon, E.-A. Kim, N. Samarth, and D. C. Ralph, Nature (London) 511, 449 (2014).

[12] S. V. Eremeev, Yu. M. Koroteev and E. V. Chulkov, JETP Lett 91, 387 (2010).

[13] M. G. Vergniory, T. V. Menshchikova, S. V. Eremeev, and E. V. Chulkov, JETP Lett. 95, 213 (2012).

[14] L. A. Wray, S.-Y. Xu, Y. Xia, D. Hsieh, A. V. Fedorov, Y. S. Hor, R. J. Cava, A. Bansil, H. Lin, and M. Z. Hasan, Nat. Phys. 7, 32 (2011).

[15] Y. L. Chen, J. G. Analytis, J. H. Chu, Z. K. Liu, S. K. Mo, X. L. Qi, H. J. Zhang, D. H. Lu, X. Dai, Z. Fang, S. C. Zhang, I. R. Fisher, Z. Hussain, and Z. X. Shen, Science 325, 178 (2009).

[16] T. Zhang, P. Cheng, X. Chen, J. F. Jia, X. Ma, K. He, L. Wang, H. Zhang, X. Dai, Z. Fang, X. Xie, and Q. K. Xue, Phys. Rev. Lett. 103, 266803 (2009).

[17] K. Kuroda, M. Arita, K. Miyamoto, M. Ye, J. Jiang, A. Kimura, E. E. Krasovskii, E. V. Chulkov, H. Iwasawa, T. Okuda, K. Shimada, Y. Ueda, H. Namatame, and M. Taniguchi, Phys. Rev. Lett. 105, 076802 (2010).

[18] Sumalay Roy, H. L. Meyerheim, A. Ernst, K. Mohseni, C. Tusche, M. G. Vergniory, T. V. Menshchikova, M. M. Otrokov, A. G. Ryabishchenkova, Z. S. Aliev, M. B. Babanly, K. A. Kokh, O. E. Tereshchenko, E. V. Chulkov, J. Schneider, and J. Kirschner, Phys. Rev. Lett. 113, 116802 (2014).

[19] P. Sessi, M. M. Otrokov, T. Bathon, M. G. Vergniory, S. S. Tsirkin, K. A. Kokh, O. E. Tereshchenko, E. V. Chulkov, and M. Bode, Phys. Rev. B 88, 161407 (2013).

[20] T. V. Menshchikova, M. M. Otrokov, S. S. Tsirkin, D. A. Samorokov, V. V. Bebneva, A. Ernst, V. M. Kuznetsov, and E. V. Chulkov, Nanoletters 13, 6064 (2013).

[21] M. M. Otrokov, S. D. Borisova, V. Chis, M. G. Vergniory, S. V. Eremeev, V. M. Kuznetsov, and E. V. Chulkov, JETP Lett. 96, 714 (2013).

[22] O. V. Yazyev, J. E. Moore, and S. G. Louie, Phys. Rev. Lett. 105, 266806 (2010).

[23] J. Sánchez-Barriga, A. Varykhalov, J. Braun, S.-Y. Xu, N. Alidoust, O. Kornilov, J. Minár, K. Hummer, G. Springholz, G. Bauer, R. Schumann, L. V. Yashina, H. Ebert, M. Z. Hasan, and O. Rader, Phys. Rev. X 4, 011046 (2014).
[24] S. Souma, K. Kosaka, T. Sato, M. Komatsu, A. Takayama, T. Takahashi, M. Kriener, K. Segawa, and Y. Ando, Phys. Rev. Lett. 106, 216803 (2011).

[25] A. Herdt, L. Plucinski, G. Bihlmayer, G. Mussler, S. Doring, J. Krumrain, D. Grutzmacher, S. Blugel, and C. M. Schneider, Phys. Rev. B 87, 035127 (2013).

[26] C. Pauly, G. Bihlmayer, M. Liebmann, M. Grob, A. Georgi, D. Subramaniam, M. R. Scholz, J. Sanchez-Barriga, A. Varykhalov, S. Blugel, O. Rader, and M. Morgenstern, Phys. Rev. B 86, 235106 (2012).

[27] K. Segawa, Z. Ren, S. Sasaki, T. Tsuda, S. Kuwabata, and Y. Ando, Phys. Rev. B 86, 075306 (2012).

[28] S. V. Eremeev, Y. M. Koroteev, and E. V. Chulkov, JETP Lett. 92, 161 (2010).

[29] S. V. Eremeev, G. Landolt, T. V. Menshchikova, B. Slomski, Y. M. Koroteev, Z. S. Aliev, M. B. Babanly, J. Henk, A. Ernst, L. Pattheya, A. Eich, A. A. Khajetoorians, J. Hagemeister, O. Pietzsch, J. Wiebe, R. Wiesendanger, P. M. Echenique, S. S. Tsirkin, I. R. Amiraslanov, J. H. Dil, and E. V. Chulkov, Nat. Commun. 3, 635 (2012).

[30] M. G. Vergniory, T. V. Menshchikova, S. V. Eremeev, and E. V. Chulkov, Appl. Surf. Sci. 267, 146 (2013).

[31] K. Kuroda, H. Miyahara, M. Ye, S. V. Eremeev, Yu. M. Koroteev, E. E. Krasovskii, E. V. Chulkov, S. Hiramoto, C. Moriyoshi, Y. Kuroiwa, K. Miyamoto, T. Okuda, M. Arita, K. Shimada, H. Namatame, M. Taniguchi, Y. Ueda, and A. Kimura, Phys. Rev. Lett. 108, 206803 (2012).

[32] K. Okamoto, K. Kuroda, H. Miyahara, K. Miyamoto, T. Okuda, Z. S. Aliev, M. B. Babanly, I. R. Amiraslanov, K. Shimada, H. Namatame, M. Taniguchi, D. A. Samorokov, T. V. Menshchikova, E. V. Chulkov, and A. Kimura, Phys. Rev. B 86, 195304 (2012).

[33] I. V. Silkin, T. V. Menshchikova, M. M. Otrokov, S. V. Eremeev, Yu. M. Koroteev, M. G. Vergniory, V. M. Kuznetsov, and E. V. Chulkov, JETP Lett. 96, 322 (2012).

[34] K. Miyamoto, A. Kimura, T. Okuda, H. Miyahara, K. Kuroda, H. Namatame, M. Taniguchi, S. V. Eremeev, T. V. Menshchikova, E. V. Chulkov, K. A. Kokh, and O. E. Tereshchenko, Phys. Rev. Lett. 109, 166802 (2012).

[35] L. E. Shelimova, O. G. Karpinskii, M. A. Kretova, I. V. Kosyakov, V. A. Shestakov, V. S. Zemskov, and F. A. Kuznetsov, Inorgan. Mater. 36, 768 (2000).

[36] G. Kresse and J. Hafner, Phys. Rev. B 48, 13115 (1993).

[37] G. Kresse and J. Furthmüller, Comp. Mater. Sci. 6, 15 (1996).

[38] P. E. Blöchl, Phys. Rev. B 50, 17953 (1994).

[39] G. Kresse and D. Joubert, Phys. Rev. B 59, 1758 (1999).

[40] J. P. Perdew, K. Burke, and M. Ernzerhof, Phys. Rev. Lett. 77, 3865 (1996).

[41] D. D. Koelling and B. N. Harmon, J. Phys. C. 10, 3107 (1977).

[42] S. Grimme, J. Comp. Chem. 27, 1787 (2006).

[43] T. V. Menshchikova, S. V. Eremeev, Yu. M. Koroteev, V. M. Kuznetsov, and E. V. Chulkov, JETP Lett. 93, 15 (2011).

[44] T. V. Menshchikova, S. V. Eremeev, and E. V. Chulkov, Appl. Surf. Sci. 267, 1 (2013).

[45] M. Nomura, S. Souma, A. Takayama, T. Sato, T. Takahashi, K. Eto, K. Segawa, and Y. Ando, Phys. Rev. B 89, 045134 (2014).

[46] J. Henk, A. Ernst, S. V. Eremeev, E. V. Chulkov, I. V. Maznichenko, and I. Mertig, Phys. Rev. Lett. 108, 206801 (2012). 